\title{
Brain Glia Release Factors with Opposing Actions upon Neuronal Survival
}

\author{
Dana Giulian, Ken Vaca, and Margaret Corpuz \\ Department of Neurology, Baylor College of Medicine, Houston, Texas 77031
}

\begin{abstract}
Microglia and astroglia have been thought to govern the survival of neurons after damage to the CNS. To investigate these putative glia-neuron relationships, we examined microglia and astroglia secretion products for effects upon growth of cultured neurons. Activated microglia secrete small neurotoxic factors $(<500 \mathrm{Da}$ ), while astroglia constitutively release proteins $(>10 \mathrm{kDa})$ that promote neuronal growth. Proteins released from astroglia, moreover, attenuate microglial toxicity, suggesting that different glial populations have opposing actions upon neuronal survival.

Further study shows that neurotoxins from microglia are heat-stable, protease-resistant molecules with biologic activities blocked by NMDA receptor antagonists. Microglial factors, although toxic for chick ciliary neurons and rat spinal cord neurons, did not reduce numbers of oligodendroglia, astroglia, or Schwann cells in culture. The microglial neurotoxins can be distinguished from cytokines, from free radical intermediates, from the excitatory amino acids glutamate or aspartate, and from the NMDA receptor-mediated toxin quinolinic acid. We propose that secretion products from reactive microglia, but not astroglia, endanger surviving neurons after CNS injury by release of a novel class of neuron-killing molecules.
\end{abstract}

[Key words: microglia, macrophages, inflammation, astroglia, neurons, CNS]

Damage to the CNS of mammals often leads to permanent loss of neurologic function. By supporting neuronal growth and metabolism, glial cells may determine, in part, the degree of recovery after CNS injury (Silver and Sidman, 1980; Aguayo et al., 1981; Reier et al., 1983; Giulian, 1987). Although reactive astroglia, reactive microglia, and invading blood-borne macrophages appear acutely following an insult to the CNS (RioHortega, 1932; Bignami and Dahl, 1976; Berry et al., 1983; Giulian and Robertson, 1990), it remains uncertain how these cells influence neuron survival, axonal regeneration, or recovery of neurologic function.

Astroglia have long been considered impediments to the regeneration of mammalian CNS (Berry et al., 1983; Reier et al., 1983); however, most in vitro studies show that astroglia actually

\footnotetext{
Received Mar. 31, 1992; revised June 8, 1992; June 25, 1992.

We thank Dr. Shen-Nan Lin. University of Texas at Houston, and Dr. Robert Schwarcz, University of Maryland Psychiatric Institute, for determining quinolinic acid levels. We also thank Dr. Clay Goodman, Baylor College of Medicine, for amino acid analyses, and Dr. Arlene Chiu, Beckman Research Institute, for the gift of MO3B antibody. The work was supported by funding from the American Paralysis Association and by Grants NS25637 and NS27616 from the NINDS.

Correspondence should be addressed to Dana Giulian, 1 Baylor Plaza, Department of Neurology, Baylor College of Medicine, Houston, TX 77030.

Copyright (c) 1993 Society for Neuroscience $0270-6474 / 93 / 130029-09 \$ 05.00 / 0$
}

support neuronal growth and survival (Ferrara et al., 1988; Manthorpe et al., 1989). In contrast, cell culture studies indicate that microglia release cytotoxic agents including free radicals (Giulian and Baker, 1986; Colton and Gilbert, 1987). Reactive microglia are also associated with a progressive loss of motor neurons and decline in motor function after ischemic injury to the spinal cord (Giulian and Robertson, 1990), with the dementias of Alzheimer's disease (McGeer et al., 1987) and acquired immunodeficiency syndrome (AIDS; Giulian et al., 1990), and with presence of neurotoxins after CNS trauma (Giulian, 1990). Such observations suggest that different populations of glia may control recovery of injured CNS by complex and, perhaps, conflicting actions. To explore these putative glial influences, we examined soluble factors released by astroglia and microglia for effects upon the growth and survival of neurons in vitro. As reported here, microglia secrete nonproteinaceous neuron-killing factors while astroglia release proteins that act as neuronal growth factors. Further study shows that the microglia-derived toxins represent a novel class of neuron poisons.

\section{Materials and Methods}

Glial cell culture. Mixed glial cultures were prepared from brain of neonatal rat as described earlier (Giulian et al., 1986). Astroglia and oligodendroglia were identified using indirect immunofluorescence by staining for glial fibrillary acidic protein (GFAP) or by staining for galactocerebroside (galC; Giulian et al., 1986). Highly enriched cultures of ameboid microglia were isolated by the method of Giulian and Baker (1986). Cultures of enriched astroglia (Giulian et al., 1986) were treated further with $1 \mathrm{~mm}$ L-leucine methyl ester and carbonyl iron $(1.5 \mathrm{mg}$ per $100 \mathrm{~mm}$ culture dish) to eliminate microglia. Isolated glial preparations were then seeded in $100 \mathrm{~mm}$ culture dishes at a density of $10^{6}$ cells per $\mathrm{ml}$ of chemically defined N2 medium (Bottenstein and Sato, 1979) supplemented with $10 \%$ fetal bovine serum. After $24 \mathrm{hr}$, the cells were incubated with $\mathrm{N} 2$ medium alone and harvested $72 \mathrm{hr}$ later. Zymosan A particles (Davis, $1981 ; 0.5 \mathrm{mg} / \mathrm{ml}$; Sigma) were added to some of the cultures to activate cells. Media conditioned by glia were filtered through $0.2-\mu \mathrm{m}$-pore membrane and stored at $-80^{\circ} \mathrm{C}$. Resident peritoneal macrophages were isolated by lavage from adult rats (Daems, 1980) and conditioned media prepared as described for microglia using a density of $10^{6}$ cells per $\mathrm{ml}$ of media. Microglia and macrophages were identified by ability to engulf particles and by the fluorescent marker $1,1^{\prime}$-dioctadecyl-1,-3,3,3',3'-tetramethylindocarbocyanine perchlorate (DiI) bound to acetylated low density lipoprotein (ac-LDL) (Giulian and Baker, 1985; Giulian et al., 1989).

Neuronal cell culture. Chick ciliary ganglia from 9-d-old embryos were dissociated by trituration with fire-polished Pasteur pipettes following a 9 min incubation with $0.08 \%$ trypsin (ICN) in $\mathrm{Ca}^{2+} / \mathrm{Mg}^{2+}$-free medium at $37^{\circ} \mathrm{C}$ (Vaca et al., 1989). The cells were collected by centrifugation at $600 \times g$, resuspended in $\mathrm{N} 2$ culture medium supplemented with 30 mм $\mathrm{KCl}$ and $0.4 \%$ heat-inactivated horse serum, and plated on polyL-lysine-coated glass coverslips at a density of about $2000 \mathrm{cells} / \mathrm{cm}^{2}$. After $2 \mathrm{~d}$ in culture, neurons were fixed for $2 \mathrm{hr}$ with $3 \%$ formaldehyde in phosphate-buffered saline (PBS; pH 7.2) and viewed at $200 \times$ using phase-contrast microscopy. For each cell count determination, we de- 
fined a healthy, surviving neuron as one that exhibited a distinct nuclear membrane with characteristic nucleoli and a cytoplasm free of large vacuoles. To assess neuron survival in cultures exposed to putative neurotoxic factors, the mean number of healthy cells was determined by scoring the number of neurons in at least 18 randomly selected fields for each of at least three coverslips. Untreated neuron cultures provided an internal control for each experiment. Dissociated chick ciliary ganglia consisted of nearly $50 \%$ cholinergic neurons, with the remainder Schwann cells and $<1 \%$ fibroblasts. For coculture experiments, isolated microglia or astroglia at concentrations of $10^{4}, 10^{5}$, or $10^{6}$ were transferred in 1 $\mathrm{ml}$ of $\mathrm{N} 2$ culture medium to Millicell-CM chambers $(0.4 \mu \mathrm{m}$ filter pore size; Millipore) that had been placed in 12-well plates with ciliary neurons growing on $18 \mathrm{~mm}$ round glass coverslips coated with poly-L-lysine. Cocultures were grown, fixed, and scored as described above. Fixed ciliary neurons were labeled by immunohistochemical staining using murine monoclonal antibodies for choline acetyltransferase (from Dr. Garrett Crawford, Baylor College of Medicine; ascites fluid, 1:200 dilution) or for neurofilament (antibody MO3B directed against the 160 kDa neurofilament protein from Dr. Arlene Chiu, Beckman Research Institute, City of Hope, CA) using the indirect immunoperoxidase method (ABC Kit, Dako).

Ciliary neuron cultures grown on glass coverslips were prepared for scanning EM by fixation with $2 \%$ glutaraldehyde in $50 \mathrm{~mm}$ cacodylate buffer ( $\mathrm{pH} \mathrm{7.4)} \mathrm{containing} 0.1 \mathrm{~m}$ sucrose for $30 \mathrm{~min}$ at $37^{\circ} \mathrm{C}$ and then at $4^{\circ} \mathrm{C}$ for $12 \mathrm{hr}$. After staining with $1 \%$ osmium tetroxide in $0.1 \mathrm{M}$ phosphate buffer ( $\mathrm{pH} 7.4$ ), cells were dehydrated by a graded series of ethanol washes and acetone prior to critical point drying. Gold-coated preparations were then viewed at $1000 \times$ magnification using a JOEL CX-100 electron microscope.

Spinal cords were removed from 15-d-old albino rat embryos (Holtzman, Madison, WI) and dissected free of attached meninges and ganglia. After incubation with $0.08 \%$ trypsin in $\mathrm{Ca}^{2+} / \mathrm{Mg}^{2+}$-free PBS for $10 \mathrm{~min}$ at $37^{\circ} \mathrm{C}$, the cords were transferred to $\mathrm{N} 2$ medium supplemented with $10 \%$ horse serum, and dissociated by trituration with fire-polished Pasteur pipettes. Cells were plated at a density of 500,000 cells $/ 0.5 \mathrm{ml} /$ well of a 24-well tissue culture plate (Falcon) with each well containing a poly-L-lysine-coated 12-mm-diameter glass coverslip. Twenty-four hours after plating, control or conditioned media were added and the cultures incubated another $72 \mathrm{hr}$ prior to fixation with buffered $3 \%$ formaldehyde. Fixed neurons were identified in mixed cell populations of rat spinal cords by indirect immunofluorescence staining with a 1:500 dilution of antibody MO3B followed by a 1:100 dilution of rhodamineconjugated, rabbit anti-mouse IgG (Dako). Under these conditions, control cultures contained $<5 \%$ neurofilament $(+)$ neurons.

Biochemical studies. Conditioned media were fractionated by ultrafiltration using YM-1 membranes (Amicon; manufacturer's estimated cutoff of $1000 \mathrm{Da}$ ) or YM-10 membranes (estimated cutoff of 10,000 Da). Gel filtration chromatography (BioGel-P2, $50.0 \times 0.7 \mathrm{~cm}$ ) eluted with $50 \mathrm{~mm}$ PBS was used to estimate molecular mass. Reverse-phase HPLC (RP-HPLC) chromatography was carried out under isocratic conditions (C18 Nova-Pak, $3.9 \times 50 \mathrm{~mm}$; Waters) with $10 \%$ acetonitrile containing $0.1 \%$ trifluoroacetic acid $(\mathrm{pH} \mathrm{2.2)}$ at a flow rate of $1 \mathrm{ml} / \mathrm{min}$. Amino acid concentrations were determined by Dr. Clay Goodman (Baylor College of Medicine) using a Waters PicoTag System. Quantitative measurements of quinolinic acid levels in conditioned medium concentrated with ion-exchange resin (Giulian et al., 1990) or from RPHPLC fractions were performed by Dr. Shen-Nan Lin (University of Texas at Houston) using a Finningan MAT Incos 50 gas chromatography/mass spectrometer. Concentrations of quinolinic acid were also determined by Dr. Robert Schwarcz (University of Maryland Psychiatric Institute), using a radioenzymatic assay (Foster et al., 1986).

\section{Results}

Microglia secrete factors that damage neurons

We used coculture techniques to explore what effects secretion products from specific glial populations might have upon neuronal growth and survival. Neurons from dissociated chick ciliary ganglia were grown on coverslips in culture dishes that held filtered chambers $(0.4 \mu \mathrm{m}$ pore size $)$ containing highly enriched populations of ameboid microglia ( $>98 \%$ homogeneity) or astroglia ( $>99 \%$ homogeneity). Although microglia or astroglia were not in direct contact with ciliary neurons, products from these glial cells diffused through the filter and into the culture

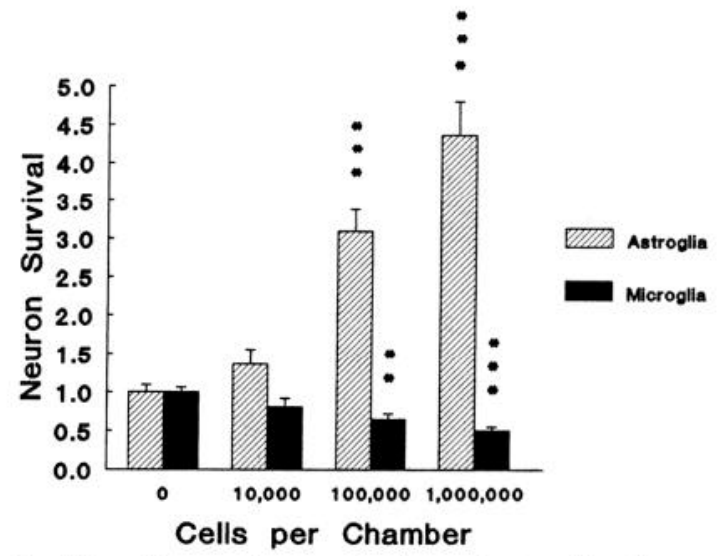

Figure 1. Microglia secrete neurotoxins and astroglia release neuron survival-promoting factors. Isolated DiI-ac-LDL $(+)$ microglia $(>98 \%$ homogeneity) or GFAP $(+)$ astroglia ( $>99 \%$ homogeneous population) were seeded at the densities indicated and placed in MilliCell-CM chambers. The chambers were then placed into wells (12-well plates; $3.8 \mathrm{~cm}^{2} /$ well) and cocultured for $48 \mathrm{hr}$ with approximately 8000 chick ciliary neurons per well grown on poly-L-lysine-coated glass coverslips. Both microglia and astroglia were incubated with zymosan A particles. Viable neurons were identified by phase-contrast microscopy at $200 \times$ magnification. Neuron survival scores (neurons per field in treated group/ neurons per field in the untreated control group) are presented as mean values \pm SE. Each value was obtained from 18 fields per coverslip using at least four coverslips per group. Zymosan A particles placed in the MilliCell-CM chambers alone did not affect neuronal survival. Significant differences among controls and microglia or astroglia cocultures are noted at 100,000 and at $1,000,000$ cell concentrations using Student's $t$ test $\left({ }^{* *}, p<0.001 ;{ }^{* * *}, p<0.0001\right)$ with a confidence level for six comparisons estimated at $p<0.008$ using the Bonferroni method (Godfrey, 1985).

medium. We observed that ameboid microglia [activated by the immunostimulant zymosan A (North, 1978; Nathan et al., 1980)] discharged agents that killed ciliary neurons while astroglia released factors promoting neuronal survival (Fig. 1). Increasing cell numbers brought about increasing amounts of biologic activity, with $>100,000$ microglia per chamber producing a reliable and significant neuronal loss of about $50 \%$.

The cytotoxic activity from microglia could be recovered in culture medium conditioned for at least $24 \mathrm{hr}$. Phase-contrast microscopy showed neurons developed vacuolated cytoplasm and lost nucleoli within $15 \mathrm{hr}$ after exposure to microglial toxins; there was widespread destruction of neurons by $48 \mathrm{hr}$ (Fig. 2). Scanning EM confirmed that microglial secretion products caused extensive neuronal damage with broken or misshapen cells and debris scattered throughout treated cultures. Monitoring neuronal survival by EM indicated a $60 \%$ loss of intact neurons, similar to that observed by phase-contrast microscopy (data not shown). This toxic effect was also demonstrated by immunohistochemistry, which revealed a significant dropout of ChAT $(+)$ or neurofilament $(+)$ ciliary neurons with remaining cells often appearing shriveled and misshapen (Fig. 3). Despite dramatic injury to neurons, the numbers and surface morphologies of Schwann cells appeared by light and electron microscopy to be unchanged in the presence of microglia-derived toxins.

\section{Different glia-derived molecules have opposing actions upon neuron survival}

Coculture experiments had shown that astroglia promoted neuronal growth (Fig. 1). This soluble biologic activity, when recovered from astroglial conditioned medium, supported the sur- 

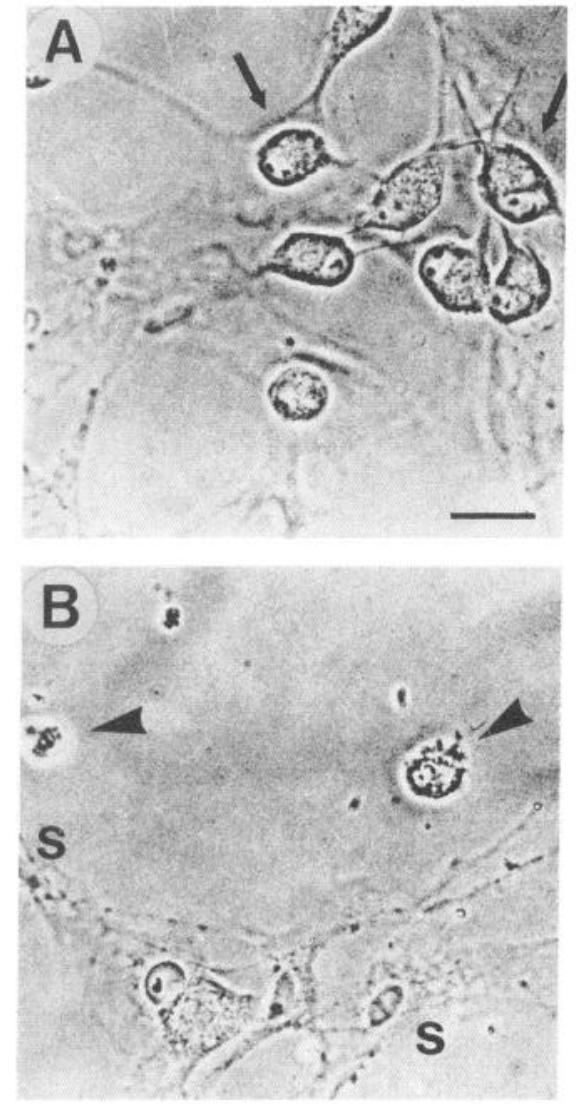

Figure 2. Phase-contrast photomicrographs showing the effects of microglial secretion products upon cultures of ciliary ganglia. As noted in control cultures $(A)$, neurons contain large nuclei with prominent nucleoli (arrows). When incubated for $48 \mathrm{hr}$ with medium conditioned by zymosan-activated microglia ( $25 \%$ concentration by volume; $B$ ), there is a marked loss of neurons. Dead neurons and debris (arrowheads) are apparent throughout the cultures with relative sparing of underlaying Schwann cells $(s)$. Scale bar, $25 \mu \mathrm{m}$.

vival of large, robust neurons with marked neuritic outgrowth (Fig. 3). In contrast to microglia-derived toxins, the release of astroglial neuron-promoting activity was constitutive and not influenced by exposure to microglial activators including zymosan particles. To delineate the character of soluble glial factors, we compared media conditioned by astroglia or microglia using ultrafiltration. Neuron-killing factors from microglia were small, protease-resistant molecules ( $<1 \mathrm{kDa}$; Fig. 4 , Table 1), whereas neuron growth-promoting factors from astroglia were much larger ( $>10 \mathrm{kDa}$, Table 1) and sensitive to trypsin degradation (Table 1). Gel chromatography showed that these astroglia-derived proteins contained several peaks of biologic activity with masses $>10 \mathrm{kDa}$ (by G-200 chromatography; K. Vaca and E. Wendt, unpublished observations). Such astroglial factors probably included ciliary neuronotrophic factor and fibroblast growth factor (Manthorpe et al., 1986; Ferrara et al., 1988; Stockli et al., 1989), which are known to promote ciliary neuron survival. Under no conditions did we detect neuron-killing activity in astroglial cultures.

Since the fate of cultured neurons could be altered by soluble agents from either astroglia or microglia, we tested what influence a combination of glial factors might have upon neuron survival. Using sufficient toxic activity to elicit near maximal killing ( $20 \%$ volume of microglial conditioned medium), we found that increasing amounts of astroglial conditioned medium

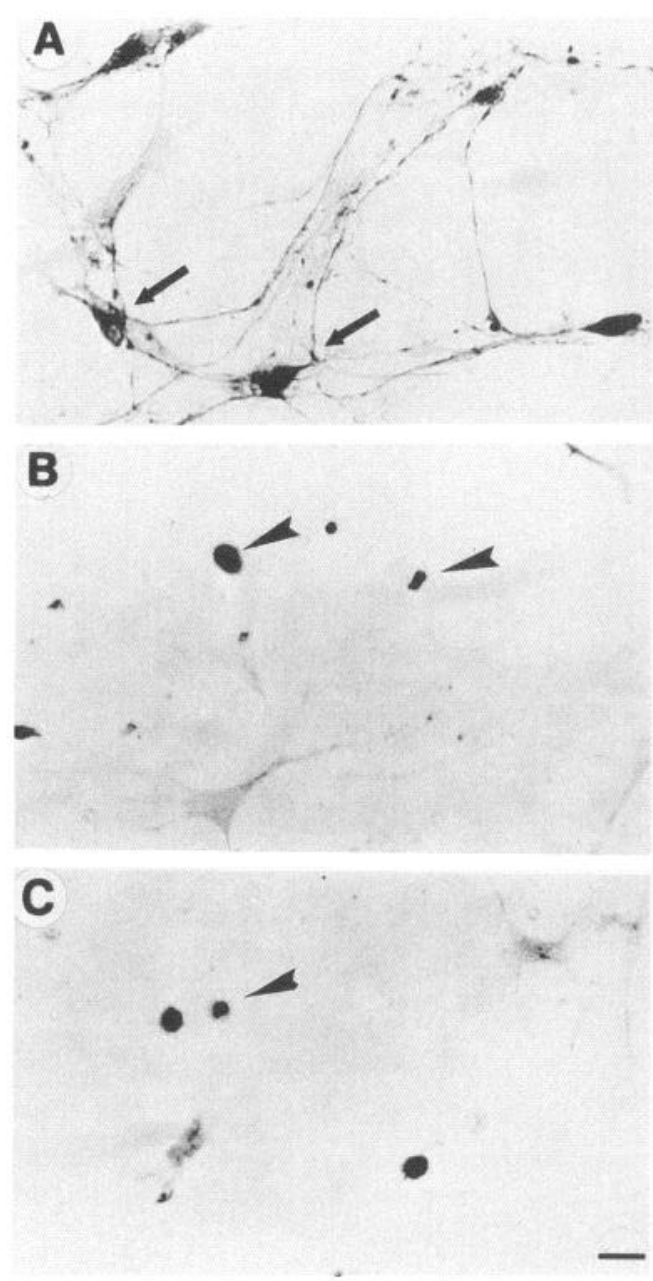

Figure 3. Light photomicrographs showing the effects of astroglial secretion products upon ciliary neurons labeled by indirect immunoperoxidase staining for neurofilament. $A$, Medium conditioned by astroglia ( $20 \%$ concentration by volume) gives rise to large, darkly stained perikaryas (arrows) and a network of delicate neurites. In contrast, cultures incubated with medium conditioned by zymosan-activated microglia $(B)$ or zymosan-activated macrophages $(C)$ had a only few, small, misshapen neurofilament $(+)$ cells that lacked neuritic outgrowth (arrowheads). Scale bar, $25 \mu \mathrm{m}$.

attenuated the toxic effects of microglia (Fig. 5). In culture, therefore, we uncover a competition between glial populations for control of neuron survival.

\section{Specificity of neuron-killing activity from microglia}

We had demonstrated so far that microglia released small molecules that were toxic to cultured chick ciliary neurons. Both light and scanning electron microscopy suggested, moreover, that neurons were the principal targets of the microglial poisons. To assess further the specificity of this cytotoxic action, we monitored survival of neurons and glia obtained from embryonic rat. Microglia-derived toxicity reduced the numbers of neurofilament $(+)$ neurons in cultures of dissociated rat spinal cord by about $50 \%$ (Table 2 ). To determine whether these neurotoxic effects were mediated in some fashion by non-neuronal support cells, we enriched ciliary neuron populations by preplating techniques (Needels et al., 1987) from about 50\% [52 $\pm 3 \%$ neurons by phase contrast; $48 \pm 1 \% \mathrm{ChAT}(+)$ cells; $52 \pm 1 \%$ neurofilament $(+)$ cells] to $95 \%$ [ $95 \pm 2 \%$ neurons by phase contrast; 

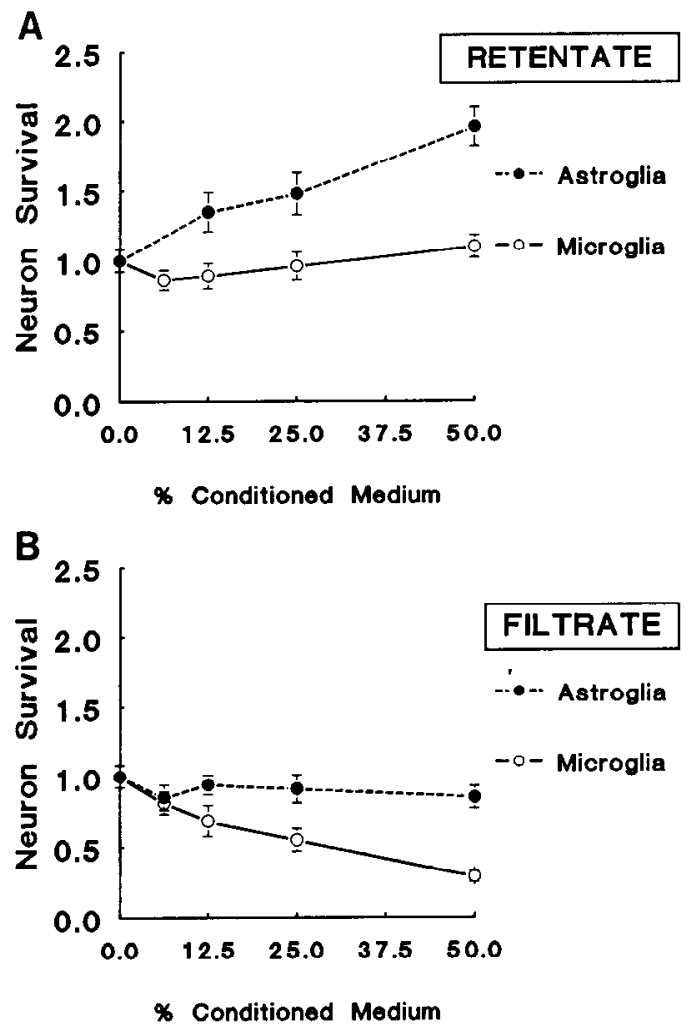

Figure 4. Dose-response curves demonstrate that different classes of glial secretion products influence the survival of ciliary neurons. $A$, Media conditioned by zymosan-activated microglia or astroglia were separated by ultrafiltration. Although astroglial factors found in the retentate $(>1 \mathrm{kDa})$ promote ciliary neuron survival, no growth-stimulating activity was produced by activated microglia. $B$, The filtrate of conditioned media shows that microglia, but not astroglia, release small molecules $(<1 \mathrm{kDa})$ that act as neuron-killing factors.

$96 \pm 2 \%$ ChAT $(+)$ cells; $92 \pm 5 \%$ neurofilament $(+)$ cells]. Microglial factors showed nearly identical toxic effects upon these two culture preparations, indicating that neuron killing was not mediated through Schwann cells (Fig. 6). Moreover, dose-response curves confirmed that microglial factors $(<1 \mathrm{kDa})$ did not alter the growth or survival of GFAP(+) astroglia, DiIac-LDL(+) microglia, or galC(+) oligodendroglia cultured from

Table 1. Properties of neuron growth-regulating factors

\begin{tabular}{llll} 
& \multicolumn{2}{l}{ Ciliary neuron survival score } \\
\cline { 2 - 4 } Treatment & Microglia & Astroglia & Macrophages \\
\hline Untreated & $0.56 \pm 0.04$ & $1.97 \pm 0.09$ & $0.59 \pm 0.04$ \\
Heat & $0.50 \pm 0.03$ & $0.95 \pm 0.04$ & $0.57 \pm 0.05$ \\
Protease & $0.51 \pm 0.04$ & $1.05 \pm 0.05$ & $0.55 \pm 0.05$
\end{tabular}

Data demonstrate properties of molecules released by microglia, astroglia, or macrophages that act upon cultured chick ciliary neurons. Media conditioned by microglia or macrophages were concentrated by lyophilization and fractionated by RP-HPLC (C18, NovaPak) using $10 \%$ acetonitrile with $0.1 \%$ trifluoroacetic acid. The astroglia-conditioned media was concentrated as a retentate using a YM10 filter. Samples were heated by boiling for $10 \mathrm{~min}$ at $\mathrm{pH} 7.4$. Protease treatments for microglial and macrophage factors involved proteinase $\mathrm{K}(1 \mu \mathrm{g} / \mathrm{ml})$ for $2 \mathrm{hr}$ at $37^{\circ} \mathrm{C}$. The enzymic reaction was stopped by filtration through Centricon 3. Astroglial retentate was incubated with trypsin $(1 \mu \mathrm{g} / \mathrm{ml})$ for $2 \mathrm{hr}$ at $37^{\circ} \mathrm{C}$. Trypsin was then inhibited with soybean trypsin inhibitor $(2 \mu \mathrm{g} / \mathrm{ml})$. Data are expressed as mean ciliary neuron survival $\pm \mathrm{SE}$, each based upon three cultures with 18 random fields scored per culture. Toxic activities from microglia and macrophages were associated with small, stable, nonproteinaceous molecules. Astroglia, in contrast, released growth-promoting proteins sensitive to boiling and trypsin.

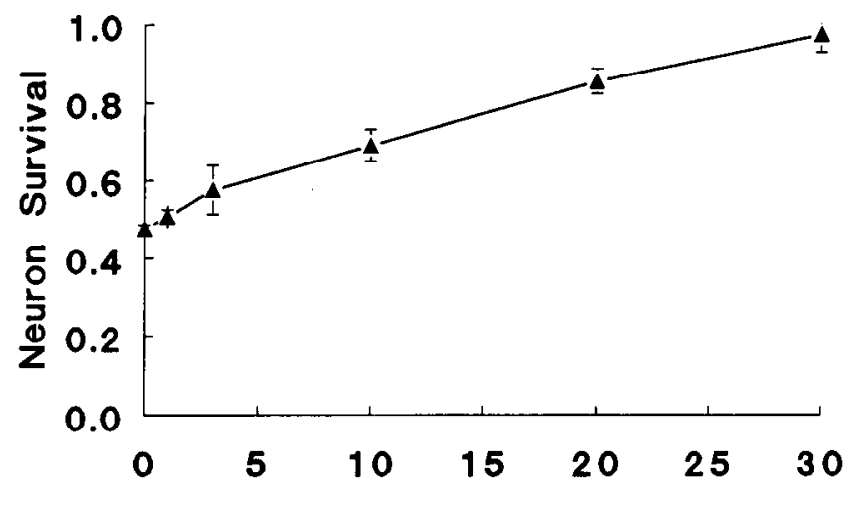

\% Astroglial Conditioned Medium

Figure 5. Opposing effects of glial secretion products upon neuronal survival. Ciliary neurons were treated with microglial toxic activity $(20 \%$ conditioned medium by volume from $10^{6}$ cells activated with zymosan for $48 \mathrm{hr}$ ) in the presence of increasing concentrations of medium conditioned by astroglia (from $10^{\circ}$ cells incubated with zymosan). As shown, astroglial factors attenuated the microglial toxins in a dose-dependent fashion. Such observations suggest that different glial populations have competing actions upon the survival of neurons.

newborn rat brains or Schwann cells obtained from chick ciliary ganglia (Fig. 7). Thus, factors released by microglia were toxic to neurons, but not glia, from the PNS or CNS.

\section{Microglial neurotoxins and inflammatory cell poisons}

Previous work has suggested that both invading macrophages and reactive microglia might produce neurotoxic molecules in response to such CNS insults as ischemia, trauma, or HIV-1 infection (Giulian, 1992). We did find that rat peritoneal macrophages released neurotoxic factors identical in action and character to those released by microglia. The macrophage-derived compounds were toxic to ciliary (Fig. 8) as well as spinal cord neurons (Table 2) and elicited a dose-dependent loss of

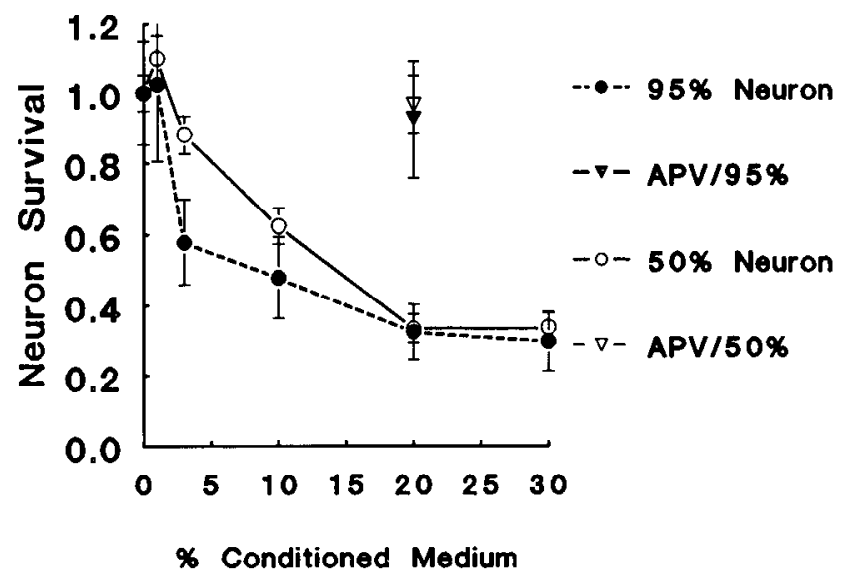

Figure 6. Microglial neurotoxicity and the influence of Schwann cells. Preplating methods allowed enrichment of ciliary neurons from a standard preparation (about 50\% neurons) to one within which about $95 \%$ of the total cell population were neurons as estimated by phase-contrast microscopy and by immunolabeling for neurofilament or ChAT. Both standard and enriched culture preparations were exposed for $48 \mathrm{hr}$ to an ultrafiltrate $(<1 \mathrm{kDa})$ of factors secreted by zymosan-activated microglia. The similarity in dose responses suggests that Schwann cells do not mediate the neurotoxic action of microglial factors. 


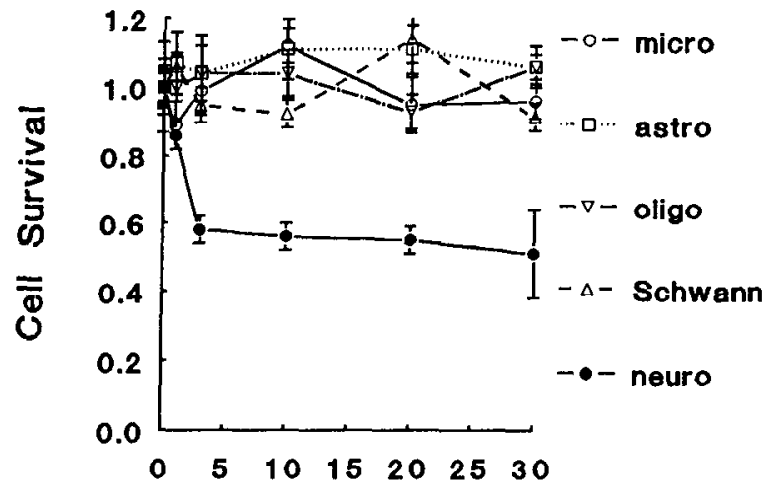

Media (\% Volume)

Figure 7. Effects of microglia-derived toxin on glia. Dose-response curvcs examined the actions of an ultrafiltrate $(<1 \mathrm{kDa})$ of secreted factors from zymosan-activated microglia upon Dil-ac-LDL $(+) \mathrm{mi}$ croglia, galC $(+)$ oligodendroglia, Schwann cells, or GFAP(+) astroglia in culture. As shown, significant cell loss was only noted for neurofilament $(+)$ ciliary neurons. Data are presented as mean values \pm SE obtained from at least nine randomly selected fields from each of three coverslips from cultures of either dissociated newborn rat brain or dissociated embryonic chick ciliary ganglia.

cells reaching about $60 \%$ after a $48 \mathrm{hr}$ incubation (Fig. 8). Both classes of mononuclear phagocytes required such activators as fixed Staphylococcus aureus or zymosan A particles (North, 1978; Nathan et al., 1980) to elicit toxin release. We did not, however, observe toxin production by either cell type after exposure to lipopolysaccharide, another class of inflammatory cell activator known to elicit cytokine release (Fig. 8; Giulian and Baker, 1986).

It is well recognized that mononuclear phagocytes release a variety of proteins $(>10 \mathrm{kDa})$ that act as cytotoxins, including tumor necrosis factor (Montgomery and Cohn, 1989), complement (Stecher and Thorbecke, 1967), interferons (Smith and Wagner, 1967), interleukins (Dinarello and Wolff, 1978), proteases (Adams, 1980), and lipases (Khoo et al., 1981). The neurotoxic activities from microglia or macrophages described here, however, were resistant to proteinase $\mathrm{K}(1 \mu \mathrm{g} / \mathrm{ml}$ for $2 \mathrm{hr}$ at $37^{\circ} \mathrm{C}$ ) and other proteases (trypsin and papain), ruling out protein cytokines and large peptides as toxic agents (Table 1). Moreover, gel filtration chromatography indicated the microglia-derived neurotoxin to be $<500 \mathrm{Da}$ (Fig. 9). There are a number of cytotoxic molecules from macrophages that have low molecular mass $(<1 \mathrm{kDa})$ such as hydrogen peroxide (Nathan and Root, 1977), nitric oxide (Liew and Cox, 1991), leukotrienes (Hoffman et al., 1987), lipoxins (Pettitt et al., 1989), and su-

\section{Table 2. Neurotoxin effects upon spinal cord neurons}

\begin{tabular}{ll} 
Media & $\begin{array}{l}\text { Spinal neuron } \\
\text { survival score }\end{array}$ \\
\hline Control & $1.00 \pm 0.06$ \\
Macrophage & $0.44 \pm 0.06^{* *}$ \\
Microglia & $0.52 \pm 0.05^{* *}$
\end{tabular}

Mononuclear phagocytes release toxins that destroy neurofilament $(+)$ rat spinal cord neurons. Cultures of dissociated embryonic rat spinal cord were incubated for $72 \mathrm{hr}$ in the presence of $50 \%$ (by volume) of media conditioned by zymosanactivated microglia or peritoneal macrophages. Values are mean scores \pm SE for neurofilament $(+)$ cells identified at $200 \times$ magnification in 18 randomly selected fields from at least five cultures per group. Significant differences are noted between control and toxin-treated groups using Student's test $\left({ }^{* *}, p<0.001\right)$.

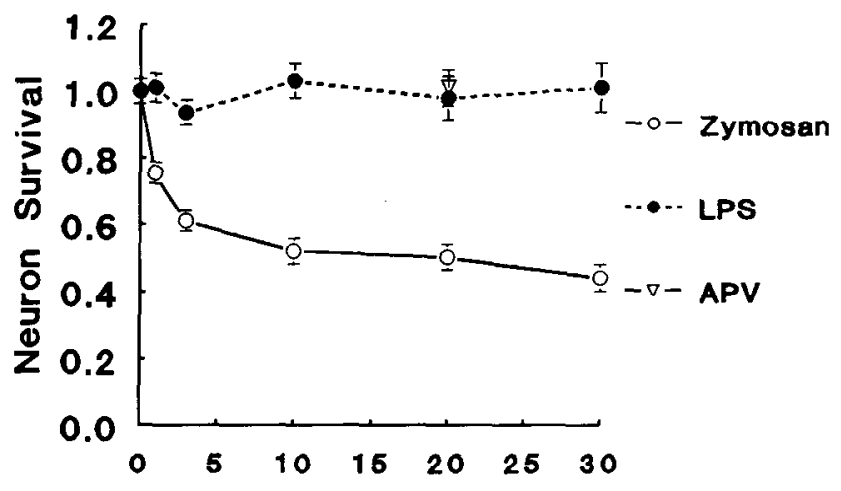

\section{\% Conditioned Medium}

Figure 8 . The toxic effects of medium conditioned by zymosan-activated macrophages. Ciliary neurons incubated for $48 \mathrm{hr}$ with increasing volumes of media from zymosan-activated macrophages showed about a maximum loss of about $50 \%$. This neurotoxic effect was blocked by the addition of $10 \mu \mathrm{M}$ APV, an NMDA antagonist. Macrophages exposed to lipopolysaccharide $(L P S ; 10 \mu \mathrm{g} / \mathrm{ml})$ did not release neurotoxic activity.

peroxide anion (Johnston et al., 1978; Giulian and Baker, 1986). However, our assay systems (which preclude cell contact and require stability in culture medium) would probably not detect such highly reactive intermediates. Moreover, the neurotoxic activity recovered from microglia or macrophages was stable to storage at $-80^{\circ} \mathrm{C}$ for at least 1 month or to boiling for $10 \mathrm{~min}$ at pH 7.4 (Table 1). For these reasons, short-lived substances such as free radicals were not directly responsible for the neuron destruction observed here. Neuron-killing activities from both activated microglia and macrophages represent, therefore, a class of heat-stable, protease-resistant, small molecules distinct from

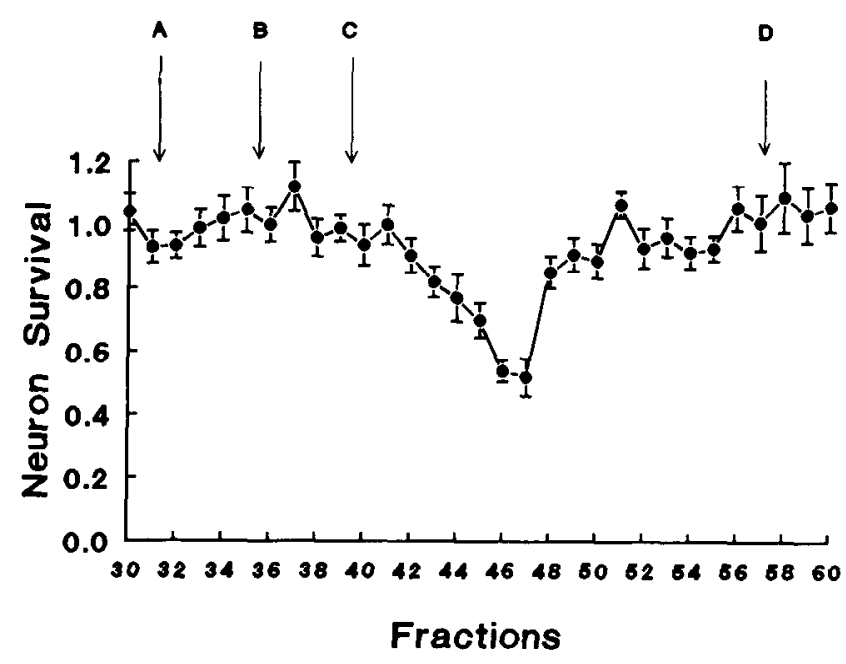

Figure 9. Neurotoxins from microglia are small molecules. Gel filtration chromatography (BioGel-P2, $50.0 \times 0.7 \mathrm{~cm}$ ) shows recovery of neuron-killing factors from media conditioned by zymosan-activated microglia with apparent molecular masses of $<500 \mathrm{Da}$. Similar elution profiles for neurotoxic activities were found in media conditioned by activated macrophages. Eluting buffer was $50 \mathrm{~mm}$ PBS with $0.30 \mathrm{ml}$ fractions collected. Each value represents a mean survival score \pm SE obtained from at least three neuron cultures. Conditioned medium was concentrated by lyophilization prior to chromatography. Experiments used ciliary neuron cultures incubated for $48 \mathrm{hr}$ with column fractions at concentrations of $2.5 \%$ by volume. Molecular mass markers: $A$, ATP, $551 \mathrm{Da} ; B, 5^{\prime}-\mathrm{GMP}, 407 \mathrm{Da}$; , cytidine, $327 \mathrm{Da} ; D$, leucine, $131 \mathrm{Da}$ 

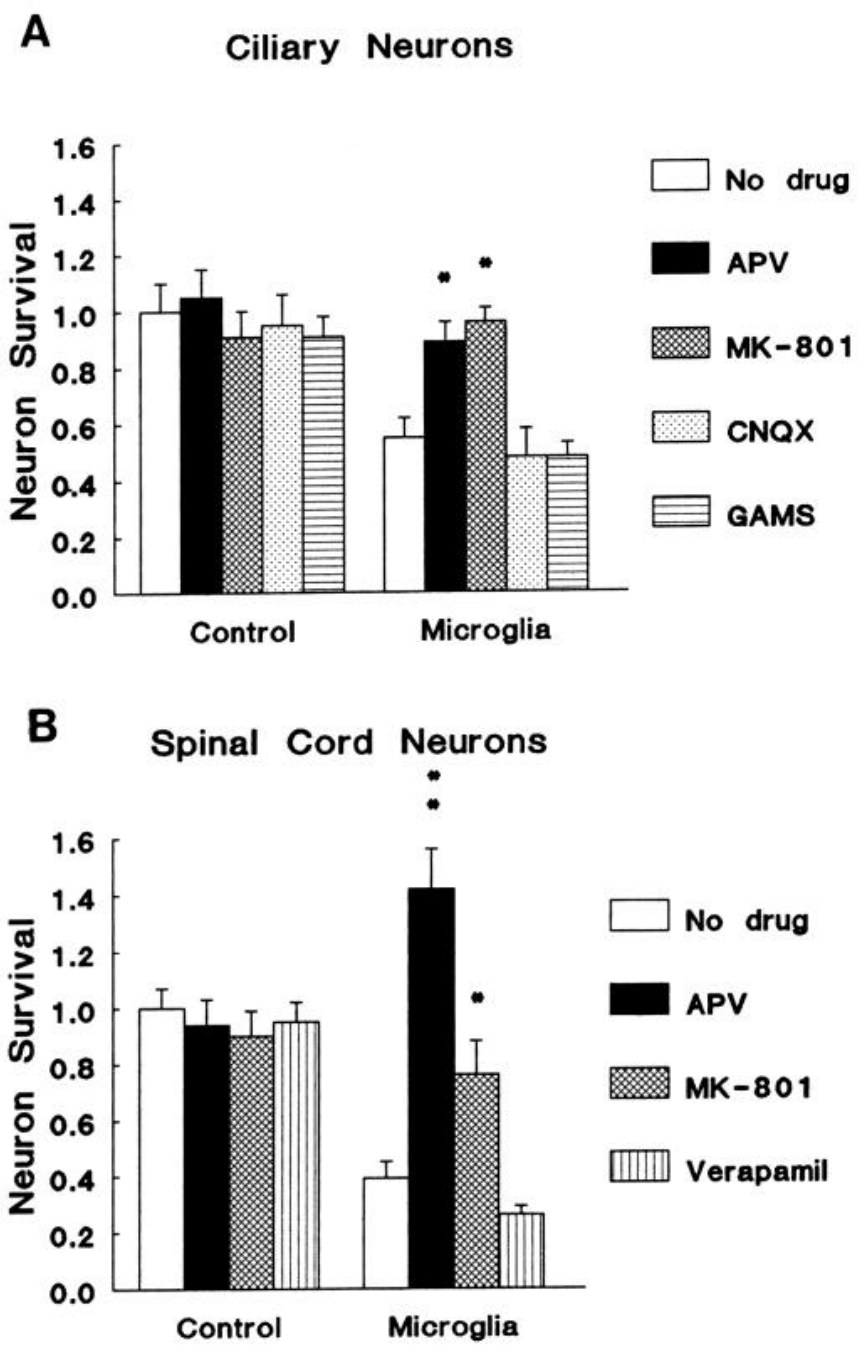

Figure 10. Blocking effects of drugs upon target activity of microgliaderived neurotoxins. Media conditioned by zymosan-stimulated ameboid microglia $\left(10^{6}\right.$ cells $/ \mathrm{ml}$ for $\left.72 \mathrm{hr}\right)$ were added to neuron cultures for $48 \mathrm{hr}$ at a concentration of $50 \%$ by volume in the presence of the indicated drugs. $A$, Neurotoxic effects of microglial-conditioned medium on ciliary neurons are blocked by the NMDA antagonist APV (10 $\mu \mathrm{M})$ and the NMDA channel blocker MK-801 (10 $\mu \mathrm{M})$. Non-NMDA receptor antagonists, CNQX $(10 \mu \mathrm{M})$ and GAMS $(10 \mu \mathrm{M})$, had no protective effect. Toxic effects are reduced significantly when compared to the no-drug cultures by Student's $t$ test $(*, p<0.01)$ with a confidence level for four comparisons estimated at $p<0.012$ using the Bonferroni method. $B$, Similarly, $10 \mu \mathrm{M}$ APV and $10 \mu \mathrm{M}$ MK-801 preserve neurofilament $(+)$ spinal cord neurons $\left({ }^{*}, p<0.01 ;{ }^{* *}, p<0.001\right)$, while the calcium L-channel antagonist verapamil $(10 \mu \mathrm{M})$ does not block the neurotoxic effect.

those previously identified as cytotoxins secreted by mononuclear phagocytes.

\section{Microglial neurotoxic effects are mediated by NMDA receptors}

Neurotoxins that are endogenous to mammalian brain include excitatory amino acids and their metabolites (Olney et al., 1974, 1990; Schwarcz et al., 1983; Honore, 1989). Acting through the NMDA class of glutamate receptor, some of these agents may be responsible for CNS damage in a variety of injuries including stroke and trauma (Rothman, 1984; Simon et al., 1984; Meldrum, 1985; Dingledine, 1986; Choi, 1988). We found that several NMDA receptor-mediated toxins including quinolinic acid
Table 3. NMDA receptor agonists as toxic molecules

\begin{tabular}{lll} 
& \multicolumn{2}{l}{ Ciliary neuron survival score } \\
\cline { 2 - 3 } Treatment & -APV & + APV $(10 \mu \mathrm{M})$ \\
\hline $\begin{array}{l}\text { Quinolinic acid } \\
100 \mathrm{nM}\end{array}$ & $0.41 \pm 0.07$ & $0.91 \pm 0.04$ \\
$1 \mu \mathrm{M}$ & $0.41 \pm 0.03$ & $0.92 \pm 0.05$ \\
AMAA & & \\
$100 \mathrm{nM}$ & $0.51 \pm 0.03$ & $0.97 \pm 0.07$ \\
$1 \mu \mathrm{M}$ & $0.48 \pm 0.06$ & $1.08 \pm 0.08$ \\
$\begin{array}{l}\text { Glutamate } \\
10 \mathrm{mM}\end{array}$ & $0.94 \pm 0.04$ & $0.93 \pm 0.04$ \\
Aspartate & & \\
$10 \mathrm{mM}$ & $1.00 \pm 0.05$ & $0.97 \pm 0.04$
\end{tabular}

Ciliary neuron cultures are sensitive to the neuron-killing effects of the NMDA toxins quinolinic acid, and AMAA in nanomolar concentrations. Receptor selectivity is indicated by the protective effect of the NMDA antagonist APV. In contrast, the excitatory amino acids glutamate and aspartate are not toxic to ciliary neurons in millimolar concentrations. Values are mean survival scores $\pm \mathrm{SE}$. Neurons were incubated with drugs for $48 \mathrm{hr}$.

and amino-3-hydroxy-5-methyl-4-isoxazoleacetic acid (AMAA) killed neurons in ciliary ganglia cultures (Table 3 ). The selective NMDA antagonist 2-amino-5-phosphonovaleric acid (APV; Honore, 1989) protected against the neurotoxicity secreted by microglia, whereas the antagonists to non-NMDA type excitatory amino acid receptors [6-cyano-7-nitroquinoxaline-2,3dione (CNQX) and $\gamma$-D-glutamylaminomethylsulfonic acid (GAMS)] had no protective effect (Fig. 10A). Similar results were obtained with rat spinal cord neurons in which APV prevented neuron killing (Fig. 10B). The NMDA receptor ion channel blocker MK-801 also protected neurons, thus confirming the involvement of the NMDA receptor-ionophore complex (Fig. $10 A$ ). In contrast, the calcium L-channel blocker verapamil did not prevent cell death (Fig. 10B). The toxicity produced by microglia and macrophages exhibited the same pharmacological properties including the protective effects of APV (Fig. 8) and MK-801.

To determine whether inflammatory cell-derived neurotoxins were, in fact, known NMDA-mediated toxins, we partially purified cell-killing activity from Hanks' buffered saline that had been conditioned by zymosan-stimulated microglia or macrophages over a $24 \mathrm{hr}$ period. A two-step purification, using ultrafiltration and gel filtration chromatography, yielded neurotoxic activity from either microglia or macrophages that sharply eluted on an RP-HPLC column (Fig. 11A). Importantly, this toxicity did not copurify with quinolinic acid, glutamic acid, or aspartic acid (Fig. $11 B$ ). Moreover, analyses of the toxin-containing fractions showed very low concentrations of amino acids (Table 4). Quinolinic acid values were below the level of detection by either mass spectrometry or enzymic assay $(<2 \mu \mathrm{M}$; data not shown) and would be present in concentrations below the range of toxicity to cultured ciliary neurons. Although the composition of the neuron-killing activity derived from microglia or macrophages is unknown, we have ruled out such agents as the cytokines interleukin-1 $\alpha$ (IL- $1 \alpha$ ), IL- $1 \beta$, and tumor necrosis factor $\alpha$ (TNF $\alpha$ ), lactic acid, proteases, or free radicals.

\section{Discussion}

Reactive gliosis, a hallmark of injury to the mammalian brain, is thought to impair recovery of neurologic function (Aguayo et al., 1981; Reier, 1983). To distinguish the influences of specific 

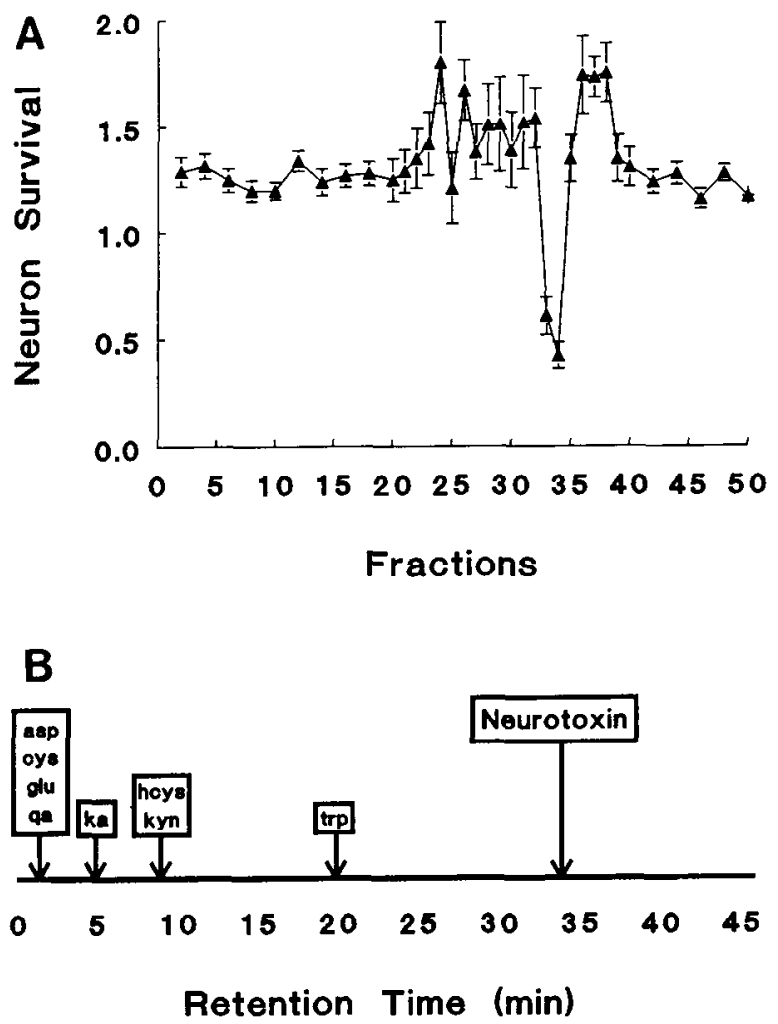

Figure 11. Fractionation of neurotoxic activity by RP-HPLC. Hanks' buffered saline conditioned for $24 \mathrm{hr}$ by zymosan-activated macrophages was recovered as an ultrafiltrate $(<1 \mathrm{kDa})$, eluted twice on a C18 column ( $10 \%$ acetonitrile with $0.1 \%$ trifluoroacetic acid at $1 \mathrm{ml} /$ inin), and recovered in $1 \mathrm{ml}$ fractions. $A$, A sharp trough of neurotoxic activity appears in fractions 34 and 35 . An identical profile was noted for the neurotoxic activity secreted by microglia. Values are expressed as mean percentage ciliary neuron survival \pm SE based upon $48 \mathrm{hr}$ assays. $B$, The macrophage-derived or microglia-derived neurotoxins can be separated from aspartic acid (asp), cysteate (cys), glutamic acid $(g / u)$, quinolinic acid $(q a)$, kynurenic acid $(k a)$, homocysteate $(h c y s)$, kynurenine $(k y n)$, and tryptophan $(t r p)$. Values are expressed as mean retention times in minutes for flow rates of $1 \mathrm{ml} / \mathrm{min}$

Table 4. Amino acid composition of neurotoxic activity

\begin{tabular}{lcc} 
& RP-HPLC fractions & $\begin{array}{c}\text { Culture } \\
\text { medium }\end{array}$ \\
\hline Glutamate & 1 & 50 \\
Aspartate & $<1$ & 50 \\
Cysteine & 5 & 0 \\
Cystine & $<1$ & 100 \\
Serine & $<1$ & 250 \\
Threonine & 4 & 400 \\
Tryptophan & 1 & 40 \\
Glycine & $<1$ & 250 \\
Glutamine & 1 & 2270 \\
Histidine & $<1$ & 550 \\
Proline & 1 & 50 \\
Leucine & $<1$ & 450
\end{tabular}

Data show amino acid composition (in $\mu \mathrm{M} /$ /iter) of neurotoxic activity recovered from RP-HPLC (fractions from C18 column as described in Fig. 11). The concentrations of amino acids in the toxin-containing fractions were generally far below that found in the culture medium used to grow neurons. Moreover, the toxin-containing fractions were tested at dilutions of 200 -fold giving final concentrations of amino acids in the range of $<5 \mathrm{~nm} /$ liter.

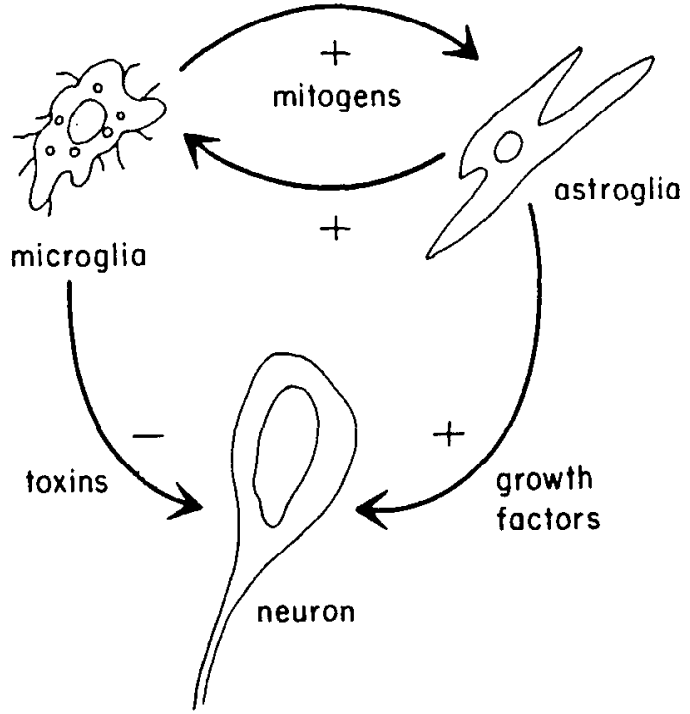

Figure 12. Glia secretion products as regulators of neuron survival. When stimulated, microglia release a variety of cytotoxic agents including the small heat-stable neurotoxic molecules described here. Similar factors that have been recovered from damaged neural tissues (Giulian, 1990) as well as HIV-I-infected monocytes (Giulian et al., 1990) may be important mediators of neuronal injury in such brain disorders as trauma, stroke, and AIDS encephalitis (Giulian, 1992). In contrast, astroglia produce a number of proteins now known to promote neuronal growth (Manthorpe et al., 1986; Ferrara et al., 1988; Vaca and Wendt, unpublished observations). Astroglia-derived growth factors attenuate the toxic effects of microglia and may help to preserve neurons under attack by inflammatory cells (Fig. 5). A further level of complexity exists for microglia release astroglial mitogens (Giulian and Baker, 1985; Giulian and Lachman, 1985; Giulian et al., 1988) while astroglia produce growth factors that stimulate microgliosis (Giulian and Ingeman, 1988; Hao et al., 1990; Malipiero et al., 1990). Ultimately, recovery of neurological function may rely upon reactive glia as they compete to govern the survival of neighboring neurons.

classes of glia during CNS injury, we monitored secretion products from astroglia and microglia for their effects upon cultured neurons. We find contrasting glial influences, with growth-promoting proteins released by astroglia and small neurotoxic molecules secreted by microglia (Giulian, 1990; Giulian et al., 1990). Such neurotoxic factors may, in turn, have in vivo significance, for several lines of evidence now suggest that mononuclear phagocytes actually engage in neuron-killing behavior. First, reactive microglia are at times found in areas of damaged tissue prior to the death of neurons (Giulian and Robertson, 1990; Gehrmann et al., 1992). Second, neurotoxic factors (identical to those reported here) have been isolated from damaged CNS (Giulian, 1990, 1992). Finally, drug suppression of reactive mononuclear phagocytes reduced motor neuron death and improved functional recovery after ischemic injury to the rabbit spinal cord (Giulian and Robertson, 1990).

Macrophages secrete a number of agents that under certain conditions scrve as cytotoxins (Nathan ct al., 1980). As noted, some of these factors may also be released by microglia and act as neurotoxins during the course of CNS injury. One of the first microglial toxins to be identified was superoxide anion (Giulian and Baker, 1986; Colton and Gilbert, 1987). More recent work (Thery et al., 1991) has confirmed microglial release of this free radical and its involvement in neuron killing when inflammatory cells are in direct contact with neurons. Other short-lived molecules released by mononuclear phagocytes may also take 
part in contact killing (Giulian, 1992). In addition, long-lived neurotoxic agents have been recovered from $\mathrm{HIV}$-1-infected monocytoid cells (Giulian et al., 1990; Pulliam et al., 1991). The toxic factors recovered from human cells remain unidentified but show similar physical properties to the rat microglial factors described here (Giulian and Noonan, 1992). Such agents would be capable of acting at a distance and could account for the loss of cortical neurons noted with AIDS (Wiley et al., 1991). Recently, Piani et al. (1991) have reported that cultured microglia constitutively release glutamate, which under some in vitro conditions is toxic to neurons. Their report is in contrast to our earlier findings on HIV-1-infected cell lines (Giulian et al., 1990) and with the data reported here, for we do not measure significant release of glutamate by activated mononuclear cells (Table 4). Second, any small change in glutamate levels would be masked by the high concentrations of the amino acid normally found in the chemically defined culture media (Table 4). More importantly, glutamate is not toxic in the ciliary neuron assay (Table 1), nor does it copurify with the macrophage- or microglia-derived neurotoxins (Fig. 11). Further study will be needed to determine if cell viability and culture conditions influence the levels of glutamate released by microglia.

The mechanisms that link astroglia, microglia, and neurons are doubtlessly complex (Fig. 12). Unopposed effects of neurotoxins from microglia during the acute phase of inflammation might limit function or survival of neurons; this neuron-killing action could, in turn, be balanced by growth factors released from astroglia at a later phase of wound repair (Reier et al., 1983; Giulian, 1992). Ultimately, glial-dependent recovery of neuronal function would rely upon the location and numbers of reactive cells associated with damaged tissue. The regulation of glial effects upon neurons is further complicated by microgliaastroglia interactions. As shown previously, microglia release astroglia-promoting growth factors (Giulian and Baker, 1985) including IL-1 (Giulian and Lachman, 1985). Such astroglial growth factors, in turn, influcnce astroglial production of such neuronal growth factors as NGF (Gadient et al., 1990; Fig. 12). Moreover, astroglia stimulate microglial growth by release of various protein mitogens (Giulian et al., 1990; Hao et al., 1990; Malipiero et al., 1990). In this way, opposing actions of secreted factors from glia might regulate neuronal survival well beyond the period of initial tissue insult.

It is unclear what signals within the brain activate microglia to release neuron-killing factors (Giulian, 1992). Cytokines may be involved, for systemic injections of TNF $\alpha$ stimulate the appearance of reactive microglia in vivo (Hickey, 1991). Similar observations have been made after intracerebral infusions of colony-stimulating factors (Giulian and Ingeman, 1988). However, as noted by Rio-Hortega (1932), necrotic tissue and hemorrhage elicit aggressive brain phagocytes and are among the most potent stimuli for reactive microgliosis. Our limited in vitro experience is consistent with this pattern in that phagocytosis-dependent cell stimuli (fixed $S$. aureus or yeast wall particles of zymosan A) are potent activators of neurotoxin release. Clearly, the type and degree of microglial stimulation would determine, in part, the level of neurotoxin production in tissues.

We have yet to identify the long-lived neurotoxic factors produced by microglia. However, these molecules can be distinguished by molecular mass and stability, by reverse-phase chromatography, and by cytotoxic potency from a number of substances described either as neuron-killing factors endogenous to the brain or as cell poisons secreted by macrophages. The lack of toxicity for excitatory amino acids in our culture systems argues against a primary effect of glutamate or aspartate. Although NMDA receptor-channel antagonists clearly attenuate the effects of microglial neurotoxins, these observations do not establish that these neurotoxins bind directly to the NMDA receptor or channel. For such reasons, the purification of microglial neurotoxins will be necessary to uncover their precise mechanisms of action.

Reactive microglia appear in almost every type of CNS disorder including infection, trauma, stroke, degeneration, and demyelination (Rio-Hortega, 1932). These inflammatory cells are the major source of CNS-derived cytokines, help to regulate wound healing in neural tissues, and scrve as an important link between the brain and systemic immune responses (Giulian, 1992). Elimination of damaged circuits would prevent continuing disruption and, perhaps, dysfunction of the entire neural system. Controlled cell death as noted after infection, neoplasm, or trauma is a well-recognized process during wound healing and tissue renewal and arises when inflammatory cells release cytotoxins. The removal of abnormal cells may be an important function for microglia during CNS inflammation (Rio-Hortega, 1932; Giulian, 1992). As sentinels of the nervous system, microglia may therefore protect healthy neurons by the destruction and rapid removal of diseased tissues. The inflammatory mechanisms responsible for disrupting function in AIDS encephalopathy and in Alzheimer's disease may be similar to the microglia-driven processes occurring after stroke and trauma. We believe that the release of long-lived neurotoxic molecules from reactive microglia represents an important neuropathic mechanism common to a variety of disease states.

\section{References}

Adams DO (1980) Effector mechanisms of cytolytically activated macrophages. I. Secretion of neutral proteases and effect of protease inhibitors. J Immunol 124:286-292.

Aguayo AJ, David S, Bray GM (1981) Influences of the glial environment on the elongation of axons after injury: transplantation studies in adult rodents. J Exp Biol 95:231-240.

Berry M, Maxwell WL, Logan A, Mathewson A, McConnell P, Ashhurst DE, Thomas GH (1983) Deposition of scar tissue in the central nervous system. Acta Neurochir [Suppl] 32:31-53.

Bignami A, Dahl D (1976) The astroglial response to stabbing. Immunofluorescence studies with antibodies to astrocyte-specific protein (GFA) in mammalian and submammalian vertebrates. Neuropathol Appl Neurobiol 2:99-1 10

Bottenstein JE, Sato GH (1979) Growth of a rat neuroblastoma cell line in serum-free supplemented medium. Proc Natl Acad Sci USA $76: 514-517$.

Choi DW (1988) Glutamate neurotoxicity and diseases of the nervous system. Neuron 1:623-634.

Colton CA, Gilbert DL (1987) Production of superoxide anion by a CNS macrophage, the microglia. FEBS Lett 223:284-288.

Daems, WT (1980) Peritoneal macrophages. In: The reticuloendothelial system (Carr I, Daems WT, eds), pp 57-127. New York: Plenum.

Davis $P$ (1981) Secretory functions of mononuclear phagocytes: overview and methods for preparing conditioned supernatants. In: Methods for studying mononuclear phagocytes (Adams DO, Edelson PJ, Koren HS, eds), pp 549-559. New York: Academic.

Dinarello CA, Wolff SM (1978) Pathogenesis of fever in man. N Engl J Med 298:607-612

Dingledine R (1986) NMDA receptors: what do they do? Trends Neurosci $9: 47-49$.

Ferrara N, Ousley F, Gospodarowicz D (1988) Bovine brain astrocytes express basic fibroblast growth factor, a neurotropic and angiogenic mitogen. Brain Res 462:223-232.

Foster AC, Okuno E, Brougher DS, Schwarcz R (1986) A radioenzymatic assay for quinolinic acid. Ann Biochem 158:98-103. 
Gadient RA, Cron KC, Otten U (1990) Interleukin-1 and tumor necrosis factor-alpha synergistically stimulate nerve growth factor (NGF) release from cultured rat astrocytes. Neurosci Lett 117:335-340.

Gehrmann J, Bonnekoh P, Miyazawa T, Hossmann KA, Kreutzberg GW (1992) Immunocytochemical study of an early microglial activation in ischemia. J Cereb Blood Flow Metab 12:257-269.

Giulian D (1987) Ameboid microglia as effectors of inflammation in the central nervous system. J Neurosci Res 18:155-171.

Giulian D, Ingeman JE (1988) Colony-stimulating factors as promoters of ameloid microglia. J Neurosci 8:4707-4717.

Giulian D (1990) Microglia, cytokines, and cytotoxins: modulators of cellular responses after injury to the central nervous system. J Immunol Immunopharmacol 10:15-21.

Giulian D (1992) Microglia and disease of the nervous system. Curr Top Neurol 12:23-54.

Giulian D, Baker TJ (1985) Peptides released by ameboid microglia regulate astroglial proliferation. J Cell Biol 101:2411-2415.

Giulian D, Baker TJ (1986) Characterization of ameboid microglia isolated from developing mammalian brain. J Neurosci 6:2163-2178.

Giulian D, Lachman LB (1985) Interleukin- I stimulation of astroglial prolifcration after brain injury. Science 228:497-499.

Giulian D, Noonan C (1992) Neurotoxins and the dementia of AIDS AIDS Res Rev 2:157-170.

Giulian D, Robertson C (1990) Inhibition of mononuclear phagocytes reduces ischemic injury in the spinal cord. Ann Neurol 27:33-42.

Giulian D, Baker TJ, Shin L-N, Lachman LB (1986) Interleukin-1 of the central nervous system is produced by ameboid microglia. J Exp Med 164:594-604.

Giulian D, Woodward J, Young D, Krebs JF, Lachman LB (1988) Interleukin-1 injected into mammalian brain stimulates astrogliosis and neovascularization. J Neurosci 8:2485-2490.

Giulian D, Chen J, Ingeman JE, George J, Noponen M (1989) The role of mononuclear phagocytes in wound healing after traumatic injury to adult mammalian brain. J Neurosci 9:4416-4429.

Giulian D, Vaca K, Noonan C (1990) Sccretion of ncurotoxins by mononuclear phagocytes infected with HIV-1. Science 250:1593-1596.

Godfrey K (1985) Comparing the means of several groups. N Engl J Med 313:1450-1456.

Hao C, Guilber LJ, FedoroffS (1990) Production of colony-stimulating factor-1 (CSF-1) by mouse astroglia in vitro. J Neurosci Res 27:314323.

Hickey WS (1991) T-Cell entry and antigen recognition in the central nervous system. In: Psycho-neuroimmunology (Adair R, Felton D, Cohen N, eds), pp 149-175. New York: Academic.

Hoffman T, Lizzio EL, Ting A, Marshall LA, Bonvini E, Jennings MK (1987) Release of arachidonic acid metabolites by human monocytes or lymphocytes: effect of treatment with interferon on stimulation by phorbol ester or calcium ionophore. Clin Immunol Immunopathol 44:82-92.

Honore T (1989) Excitatory amino acid receptor subtypes and specific antagonists. Med Res Rev 9:1-23.

Johnston RB, Godzik CA, Cohn ZA (1978) Increased superoxide anion production by immunologically activated and chemically elicited macrophages. J Exp Med 148:115-127.

Khoo JC, Mahoney EM, Witztum JL (1981) Secretion of lipoprotein lipase by macrophages in culture. J Biol Chem 256:7105-7108.

Liew FY, Cox FE (1991) Nonspecific defence mechanism: the role of nitric oxide. Immunol Today 12:A17-A21.

Malipiero UV, Frei K, Fontana A (1990) Production of hemopoietic colony-stimulating factors by astrocytes. J Immunol 144:3816-3821.

Manthorpe M, Rudge JS, Varon S (1986) Astroglial cell contributions to neuronal survival and neuritic growth. In: Astrocytes, Vol 2 (Federoff S, Vernadakis A, eds), pp 315-376. New York: Academic.

Manthorpe M, Ray J, Pettmann B, Varon S (1989) Ciliary neuronotrophic factors. In: Nerve growth factors (Rush RA, ed), pp 31-56. New York: Wiley.

McGeer PL, Itagaki S, Tago H, McGeer EG (1987) Reactive microglia in patients with senile dementia of the Alzheimer type are positive for the histocompatibility glycoprotein HLA-DR. Neurosci Lett 79: 195-200.

Meldrum B (1985) Excitatory amino acids and anoxic/ischemic brain damage. Trends Neurosci 8:47-48

Montgomery RR, Cohn ZA (1989) Endocytic and secretory repertoire of the lipid-loaded macrophage. J Leukocyte Biol 45:129-138.

Nathan C, Murray H, Cohn Z (1980) The macrophage as an effector cell. N Engl J Med 303:622-625.

Nathan CF, Root RK (1977) Hydrogen peroxide release from mouse peritoneal macrophages. J Exp Med 146:1648-1662.

Needels DL, Nieto-Sampedro M, Cotman CW (1987) Long-term support by injured brain extract of a sub-population of ciliary ganglion neurons purified by differential adhesion. Neurochem Res 12:901907.

North RJ (1978) Concept of the activated macrophage. J Immunol 121:806-809.

Olney JW, Rhee V, Ho OL (1974) Kainic acid: a powerful neurotoxic analogue of glutamate. Brain Res 77:507-512.

Pettitt TR, Rowley AF, Secombes CJ (1989) Lipoxins are major lipoxygenase products of rainbow trout macrophages. FEBS Lett 259: $168-170$.

Piani D, Frei K, Do K, Cuenod M, Fontana A (1991) Murine brain macrophages induce NMDA receptor mediated neurotoxicity in vitro by secreting glutamate. Neurosci Lett 133:159-162.

Pulliam L, Herndier BG, Tang NM, McGrath MS (1991) Human immunodeficiency virus-infected macrophages produce soluble factors that cause histological and neurochemical alterations in cultured human brains. J Clin Invest 87:503-512.

Reier PJ, Stenasaas LJ, Guth L (1983) The astrocytic scar as an impediment to regeneration in the central nervous system. In: Spinal cord regeneration (Kao CC, Bunge RP, Reier PJ, eds), pp 163-195. New York: Raven

Rio-Hortega P (1932) Microglia. In: Cytology and cellular pathology of the nervous system (Penfield W, ed), pp 481-584. New York: Hocker.

Rothman S (1984) Synaptic release of excitatory amino acid neurotransmitter mediates anoxic neuronal death. J Neurosci 4:1884-1891.

Schwarcz R, Whetsell WO Jr, Mangano RM (1983) Quinolinic acid: an endogenous metabolite that produces axon-sparing lesions in rat brain. Science 219:316-318.

Silver J, Sidman RL (1980) A mechanism for the guidance and topographic patterning of retinal ganglion cell axons. J Comp Neurol 189:101-111.

Simon RP, Swan JH, Griffiths T, Meldrum BS (1984) Blockade of $N$. methyl-D-aspartate receptors may protect against ischemic damage in the brain. Science 226:850-852.

Smith TJ, Wagner RR (1967) Rabbit macrophage interferons. I. Conditions for biosynthesis by virus-infected and uninfected cells. J Exp Med 125:559-577.

Stecher VJ, Thorbecke GJ (1967) Sites of synthesis of human serum proteins. I. Serum proteins produced by macrophages in vitro. J Immunol 99:643-652.

Stockli KA, Lottspeich F, Sendtner M, Masiakowski P, Carroll P, Gotz R, Lindholm D, Thoenen H (1989) Molecular cloning, expression and regional distribution of rat ciliary neurotrophic factor. Nature 342:920-923.

Stone T, Connick JH (1985) Quinolinic acid and other kynurenines in the central nervous system. Neuroscience 15:597-617.

Thery C, Chamak B, Mallat M (1991) Free radical killing of neurons. Eur J Neurosci 3:1155-1164.

Vaca K, Stewart SS, Appel SH (1989) Identification of basic FGF as a cholinergic growth factor in human muscle. J Neurosci Res 23:5563.

Wiley CA, Masliah E, Morey M, Lemerc C, DeTeresa R, Girafte M, Hansen L, Terry R (1991) Neocortical damage during HIV infection. Ann Neurol 29:651-657. 